\title{
KOSZUL-MORITA DUALITY
}

\author{
JOSEPH CHUANG ${ }^{1}$, ANDREY LAZAREV ${ }^{2}$ \& W.H. MANNAN ${ }^{3}$
}

\begin{abstract}
We construct a generalization of Koszul duality in the sense of Keller-Lefèvre for not necessarily augmented algebras. This duality is closely related to classical Morita duality and specializes to it in certain cases.
\end{abstract}

\section{INTRODUCTION}

Koszul duality is an anti-equivalence between certain subcategories of the derived category of a quadratic Koszul algebra $A$ and that of its Koszul dual $A^{!}$ [1]. More recently Keller and Lefèvre [4] gave a general formulation which is valid for a general augmented differential graded (dg) algebra and its dg Koszul dual (which is in that case naturally a dg coalgebra). The derived category on the dual side is that of dg comodules over this dg coalgebra; this is a derived category of the second kind in the sense of [7], in particular acyclic comodules are not necessarily regarded as trivial. In fact, in the coalgebra-comodule language the Koszul equivalence becomes covariant, but taking linear duals restores contravariance. We adopt the set-up in which the Koszul correspondence is contravariant; this allows us to replace coalgebras and comodules with the dual notions of pseudo-compact algebras and pseudo-compact modules, cf. [11] concerning these notions. This language is of course equivalent to the language of coalgebras and comodules.

It is natural to ask whether the assumption that $A$ be augmented is essential for constructing Koszul duality. One answer which completely removes this assumption is provided by the work of Positselski [7], but at a price: the Koszul dual object op. cit. is no longer a pseudo-compact dg algebra but a more general one, called a (pseudo-compact) curved dg algebra. The category of modules also needs to be appropriately modified.

We provide a different answer, which ensures that (the analogue of) the Koszul dual object is still an (ordinary, not curved) pseudo-compact dg algebra, even though the original $A$ is not necessarily augmented. It is however, required to possess a non-zero finite-dimensional $\operatorname{dg}$ module $M$ (in the case $M=\mathbf{k}$, the ground field, this reduces to the Keller-Lefèvre treatment). We prove further, that such a dg module always exists, at least if we replace $A$ by a quasi-isomorphic dg algebra.

\footnotetext{
${ }^{1}$ Department of Mathematics, City University London, London EC1V 0HB. email: j.chuang@city.ac.uk

${ }^{2}$ Mathematics and Statistics, Lancaster University, Lancaster LA1 4YF. email: a.lazarev@lancaster.ac.uk

${ }^{3}$ Mathematics and Statistics, Lancaster University, Lancaster LA1 4YF. email: wajid@mannan.info
} 
The unexpected conclusion is that the derived category of dg modules over a dg algebra is always equivalent to some derived category of pseudo-compact modules.

Conversely, we give a necessary and sufficient condition for the derived category of pseudo-compact dg modules over a pseudo-compact dg algebra to be anti-equivalent to the derived category of $\mathrm{dg}$ modules over some dg algebra. In contrast, this condition does not always hold, i.e. there are derived categories of pseudo-compact modules which are not equivalent to any derived module category.

Furthermore, the functor associating to a $\mathrm{dg} A$-module an appropriate pseudocompact module on the Koszul dual side is given as a kind of (derived) Hom into $M$. It is, thus, reminiscent of the (derived version of) the classical Morita duality $[6,5]$. It turns out that our duality could indeed be viewed as an extension of Morita duality in the case when $A$ is an ordinary finite-dimensional algebra of finite global dimension.

It seems likely that a large portion of our results could be extended to dg modules over dg categories, however we refrained from working in this generality to keep exposition simple. Related results, in the context of dg categories, are contained in the recent preprint [8]. The main difference in our approach is that the use of the reduced Hochschild complex allowed us to avoid additional assumptions present in e.g. Proposition 3.9 of op. cit.

It also is worth noting that equivalences between categories of pseudo-compact modules (phrased in the language of comodules) were studied in the work of Takeuchi [10] and so our results could be viewed as linking (derived versions of) Morita theory and Takeuchi theory.

1.1. Organization of the paper. In sections 2 through 7 we formulate and prove our main result: a Quillen anti-equivalence between categories of dg modules over a dg algebra $A$, possessing a finite-dimensional non-zero $\operatorname{dg}$ module $M$, and the category of pseudo-compact $\mathrm{dg}$ modules over a pseudo-compact dg algebra $E$, which is the reduced Hochschild complex of $A$ with coefficients in End $M$. We note that $E$ computes the derived endomorphisms of $M$ as an $A$-module, just as (for example) the corresponding unreduced complex. However we cannot replace $E$ with the corresponding unreduced complex or use some other resolution of $M$ since that will change the derived category of pseudo-compact modules. We do not have a satisfactory explanation of this striking phenomenon; perhaps it is related to the lack of an appropriate closed model category structure on all pseudo-compact dg algebras (or, equivalently, all dg coalgebras).

Our main tool in proving the mentioned Quillen equivalence is Koszul duality as developed by Positselski [7]. One minor modification that we introduce is the systematic use of (pseudo-compact) dg modules and their Maurer-Cartan twisting which might be of some independent interest. It is worth remarking that the end result does not involve curved dg algebras and modules even though Positselski's results and our use of them do.

In Section 8 we consider the question of when the category of pseudo-compact dg modules is Quillen equivalent to some (ordinary) derived category of dg modules; a particularly simple criterion is formulated in the case of an ungraded pseudocompact algebra. Finally Section 9 explains how our results essentially reduce 
to (derived) Morita duality in the case when $A$ is finite-dimensional and of finite global dimension.

\section{Formulation of the main Result}

Let $A$ be a dg algebra over a field $\mathbf{k}$ and let $M$ be a non-zero finite-dimensional (dg) module over $A$ (the adjective 'dg' will typically be omitted when applied to a module over a dg algebra). All dg algebras and modules will be cohomologically graded (so the differential will have degree 1). Recall (cf., for example [7, Theorem 8.1a]) that the category of $A$-modules has the structure of a closed model category where weak equivalences are quasi-isomorphisms and fibrations are the surjective maps. All $A$-modules are fibrant and cofibrant objects are retracts of cell $A$ modules; the latter are the $A$-modules having a filtration whose associated factors are free $A$-modules. We can form the reduced Hochschild complex of $A$ with coefficients in $\operatorname{End}(M)$ :

$$
\overline{\operatorname{Hoch}}^{n}(A, \text { End } M) \subset \operatorname{Hom}\left(A^{\otimes n}, \text { End } M\right)
$$

consisting of the reduced cochains; that is multilinear maps (over $\mathbf{k}$ ) $A^{\times n} \rightarrow$ End $M$ which vanish if any of the arguments is $1 \in A$. The complex $\overline{\operatorname{Hoch}}^{\bullet}(A$, End $M)$ is a dg algebra which we will denote by $E$.

To see that it is a dg algebra first define multiplication of a degree $n$ element $\alpha$ with a degree $m$ element $\beta$ by setting application to elements $a_{1}, \cdots, a_{n}, b_{1}, \cdots, b_{m} \in$ $A$ to be given by:

$$
(\alpha \beta)\left(a_{1}, \cdots, a_{n}, b_{1}, \cdots, b_{m}\right)=(-1)^{s t} \alpha\left(a_{1}, \cdots, a_{n}\right) \beta\left(b_{1}, \cdots, b_{m}\right)
$$

where $s$ is the sum of the degrees of the $a_{i}$ and $t$ is the degree of $\beta$. The multiplication on the right hand side of his equation is just composition in $\operatorname{End}(M)$.

Note that $E$ has a differential $d$ which is the sum of:

1) The internal differentials on the copies of $A$ in $A^{\otimes n}$,

2) The differential on $\operatorname{End}(M)$ coming from the internal differential on $M$,

3) Contractions on the copies of $A$, including the two 'end terms' represented by taking the commutator $[\delta$,$] in E$, where $\delta: A \rightarrow \operatorname{End}(M)$ is just the $A$-action on $M$.

Without these 'end terms', the differential of a reduced cochain would no longer be reduced.

Moreover, $E$ can be identified (disregarding the differential) with $\hat{T} \Sigma\left((A / \mathbf{k})^{*}\right) \otimes$ End $M$ where here and elsewhere in the paper the tensor product is understood in the appropriate completed sense. Here $\Sigma$ denotes the suspension operator, which raises the degree of each homogeneous element in a graded algebra/module. Recall from [11] that a dg algebra is pseudo-compact if it is an inverse limit of finite-dimensional dg algebras (or. equivalently, is dual to a dg coalgebra). Since $M$ is finite-dimensional, the $\mathrm{dg}$ algebra $E$ is pseudo-compact. In the case $M=\mathbf{k}$, the pseudo-compact dg algebra $E$ is also local (which is the same as saying that its dual dg coalgebra is conilpotent).

We also consider the category of left pseudo-compact (dg) modules E-mod; these are just inverse limits of finite-dimensional $E$-modules (or comodules in the 
dual setting). It has the structure of a closed model category in which the weak equivalences are strictly stronger than quasi-isomorphisms and fibrations are surjections [7, Theorem 8.2a]. All $E$-modules are fibrant and the cofibrant $E$-modules are the retracts of free $E$-modules (disregarding the differential).

Here is our main result; its proof is given is section 7, after laying the groundwork in previous sections.

Theorem 2.1. The categories $A$-mod and $(E-\bmod )^{\mathrm{op}}$ are Quillen equivalent. The functor $F: A$-mod $\rightarrow(E \text {-mod })^{\mathrm{op}}$, effecting this equivalence, associates to an $A$ module $N$ the following $E$-module:

$$
F(N)=\overline{\operatorname{Hoch}}(A, \operatorname{Hom}(N, M)),
$$

the reduced Hochschild complex of $A$ with coefficients in $\operatorname{Hom}(N, M)$.

Remark 2.2. (1) We use (2.1) to define a left $E$-action on $F(N)$, with multiplication on the right hand side of (2.1) now coming from the End $M-$ action on $\operatorname{Hom}(N, M)$.

(2) The complex $\overline{\operatorname{Hoch}}(A, \operatorname{End}(M))$ is quasi-isomorphic to the unreduced Hochschild $\operatorname{complex} \operatorname{Hoch}(A, \operatorname{End}(M))$. However these pseudo-compact dg algebras do not have equivalent derived categories of pseudo-compact modules. For example, taking $A=\mathbf{k}$ and $M=\mathbf{k}$ we have $\operatorname{Hoch}(A, \mathbf{k}) \cong \overline{\operatorname{Hoch}}(A \times \mathbf{k}, \mathbf{k})$; thus the category of pseudo-compact $\operatorname{Hoch}(A, \mathbf{k})$-modules is Quillen equivalent to the category of $(\mathbf{k} \times \mathbf{k})$-modules. On the other hand, $\overline{\operatorname{Hoch}}(A, \mathbf{k}) \cong$ $\mathbf{k}$. Thus, the derived categories of pseudo-compact $\overline{\operatorname{Hoch}}(A, \mathbf{k})$-modules and of pseudo-compact $\operatorname{Hoch}(A, \mathbf{k})$-modules cannot be equivalent and the reduced Hochschild complex cannot be replaced with the unreduced one in the definition of the pseudo-compact dg algebra $E$.

(3) It is possible to describe the adjoint functor $G$ : $(E \text {-mod })^{\text {op }} \rightarrow A$-mod explicitly (and this will be done later). However for this we need to develop the language of twistings and it will be in less traditional terms than the functor $F$.

If the $A$-module $M$ is the ground field $\mathbf{k}$ (so that $A$ is augmented) then Theorem 2.1 is the ordinary dg Koszul duality. However we stress that $M$ could be an arbitrary non-zero finite-dimensional $A$-module; in particular it could be acyclic. This leads to the following result.

Corollary 2.3. For any $d g$ algebra A the category A-mod is Quillen equivalent to the category $(E \text {-mod })^{\text {op }}$ for some pseudo-compact dg algebra $E$.

Proof. Let $C$ be any finite-dimensional acyclic dg algebra; the smallest example is a two-dimensional one having basis $\{1, x\}$ with $x^{2}=0$ and $d(x)=1$. Then the projection $A \times C \rightarrow A$ is clearly a quasi-isomorphism and leads to a Quillen equivalence between the categories of $A$-modules and $A \times C$-modules. But the dg algebra $A \times C$ has a non-zero finite dimensional module, namely $C$ via the projection $A \times C \rightarrow C$. We conclude, by Theorem 2.1, that the category $A \times C$-mod and thus, the category $A$-mod, is Quillen equivalent to the category $\left(E\right.$-mod) ${ }^{\text {op }}$ where $E=\overline{\operatorname{Hoch}}(A \times C$, End $C)$. 


\section{Curved Algebras AND modules}

Recall from [7] that a curved dg algebra is a graded algebra $A$ together with a 'differential' $d: A \rightarrow A$, a derivation of $A$ of degree one such that $d^{2}(a)=[h, a]$ where $a \in A$ and $h \in A$ is an element of degree two such that $d(h)=0$, called the curvature of $A$.

A morphism of curved dg algebras $f: B \rightarrow A$ is a pair $(f, a)$ consisting of a morphism of graded algebras $f: B \rightarrow A$ and an element $a \in A^{1}$ satisfying the equations:

$$
\begin{array}{r}
f\left(d_{B}(x)\right)=d_{A}(f(x))+[a, f(x)], \\
f\left(h_{B}\right)=h_{A}+d_{A}(a)+a^{2},
\end{array}
$$

for all $x \in B$, where $B=\left(B, d_{B}, h_{B}\right)$ and $A=\left(A, d_{A}, h_{A}\right)$.

The composition of morphisms is defined by the rule $(f, a) \circ(g, b)=(f \circ g, a+$ $f(b))$. Identity morphisms are the morphisms $(i d, 0)$.

A morphism $(f, 0)$ as above is called strict; thus strict morphisms preserve curvature elements.

Note that a morphism $(f, a)$ of curved dg algebras is an isomorphism precisely when $f$ is invertible, since the inverse map is then given by the pair $\left(f^{-1},-f^{-1}(a)\right)$. Any dg algebra can be viewed as a curved dg algebra with the zero curvature. There are non-isomorphic dg algebras which are isomorphic as curved dg algebras.

A left module $\left(M, d_{M}\right)$ over a curved $\operatorname{dg}$ algebra $A$ is a graded left $A$-module M endowed with a derivation $d_{M}: M \rightarrow M$ compatible with the derivation $d_{A}$ and such that $d_{M}^{2}(x)=h_{A} x$ for any $x \in M$. Equivalently, a graded $\mathbf{k}$-vector space $M$ with degree 1 derivation $d_{M}: M \rightarrow M$ is a left $A$-module if it is equipped with a strict map $A \rightarrow$ End $M$. Here the 'differential' on End $M$ is taking the commutator with $d_{M}$ and the curvature is $d_{M}^{2}$.

Remark 3.1. A curved dg algebra $A$ is not necessarily a left (or right) module over itself. Indeed if it were, then $d_{A}^{2}=[h,-]=l_{h}$, where $l_{h}$ is the operator of left multiplication by $h$; clearly this holds if and only if $h=0$. On the other hand, it is consistent to consider $A$ as an $A$-bimodule, i.e. a module over the curved $\mathrm{dg}$ algebra $A \otimes A^{\text {op }}$ with the curvature element $h \otimes 1-1 \otimes h$.

We will be interested in the pseudo-compact versions of the above notions, i.e. pseudo-compact curved dg algebras and modules over them.

Example 3.2. Let $A$ be a unital dg algebra. Choose a $\mathbf{k}$-linear map $\epsilon: A \rightarrow \mathbf{k}$, to be regarded as an 'augmentation', even though not required to be multiplicative nor differential. Then the pseudo-compact graded algebra $\hat{T} \Sigma\left((A / \mathbf{k})^{*}\right)$ has the structure of a curved pseudo-compact algebra. To define it, view $\hat{T} \Sigma\left((A / \mathbf{k})^{*}\right)$ as a 'Hochschild complex' of $A$ with coefficients in $\mathbf{k}$. In other words, define the 'differential' on $\hat{T} \Sigma\left((A / \mathbf{k})^{*}\right)$ by the formula:

$$
\begin{aligned}
d f\left(a_{1}, \ldots, a_{n}\right) & =\epsilon\left(a_{1}\right) f\left(a_{2}, \ldots, a_{n}\right) \\
& +\sum_{k=1}^{n}(-1)^{k} f\left(a_{1}, \ldots, a_{k} a_{k+1}, \ldots, a_{n}\right) \\
& +(-1)^{n+1} f\left(a_{1}, \ldots, a_{n-1}\right) \epsilon\left(a_{n}\right)
\end{aligned}
$$


(here for simplicity it is assumed that all elements and $f$ are of even degree).

Since $\epsilon$ is not necessarily an augmentation, $d$ may not square to zero; however this will define a curved dg algebra structure on $\hat{T} \Sigma\left((A / \mathbf{k})^{*}\right)$. If $\epsilon$ is chosen to be a dg map, the curvature element is the 'homutator' of $\epsilon ; h \in \hat{T}^{2} \Sigma\left((A / \mathbf{k})^{*}\right)$ : $h(a, b)=\epsilon(a b)-\epsilon(a) \epsilon(b)$. It vanishes iff $\epsilon$ is a genuine augmentation in which case $\hat{T} \Sigma\left((A / \mathbf{k})^{*}\right)$ becomes uncurved and is isomorphic to $\overline{\operatorname{Hoch}}(A, \mathbf{k})$.

If $A$ is acyclic then one cannot choose $\epsilon$ to be a dg map. In this case the curvature element has an additional term - the differentiator $\left.h_{d} \in \Sigma(A / \mathbf{k})^{*}\right)$, satisfying $h_{d}(a)=\epsilon(d a)$.

The last example is the main reason (for us) to consider curved dg algebras. For any unital dg algebra $A$ it determines a (local) pseudo-compact algebra $\hat{T} \Sigma\left((A / \mathbf{k})^{*}\right)$. The correspondence $A \mapsto \hat{T} \Sigma\left((A / \mathbf{k})^{*}\right)$ depends on choosing a fake augmentation but any two choices are canonically isomorphic and thus, it could be viewed as a functor from dg algebras into local curved pseudo-compact dg algebras. Positselski shows that this gives a Quillen anti-equivalence between dg algebras and local pseudo-compact curved dg algebras [7]. This is a unital analogue of KellerLefevre's correspondence. There is also a Quillen anti-equivalence between $A$ modules and $\hat{T} \Sigma\left((A / \mathbf{k})^{*}\right)$-modules which will be instrumental in establishing our main result.

\section{TWIsting}

The notion of Maurer-Cartan (MC) twisting of dg algebras or dg Lie algebras is well-documented [2]. Here we will discuss twistings of curved algebras and modules over them. Note that Positselski (and others) work with twisted cochains rather than with MC elements; our point of view is essentially equivalent but more convenient since it allows one to avoid coalgebras and comodules.

Definition 4.1. (1) Let $A=(A, d, h)$ be a curved dg algebra and $\xi \in A^{1}$. The twisting of $A$ by $\xi$, denoted by $A^{\xi}$, is the curved dg algebra having the same underlying space as $A$, the twisted differential $d^{\xi}=d+\left[\xi,{ }_{-}\right]$and the twisted curvature $h^{\xi}=h+d \xi+\frac{1}{2}[\xi, \xi]$.

(2) Let $M, d_{M}$ be a module over a curved $\mathrm{dg}$ algebra $A$ as above. The twisting of $M$ by $\xi$, denoted by $M^{[\xi]}$, is the module over $A^{\xi}$ having the same underlying space as $A$ and the twisted differential $d^{[\xi]}:=d_{M}+\xi$.

Remark 4.2. An uncurved dg algebra $A$ may be viewed as a module over itself, so given $\xi \in A^{1}$ we have that $A^{[\xi]}$ is a module over $A^{\xi}$; here $A^{[\xi]} \neq A^{\xi}$ as they have different differentials.

Definition 4.3. Let $(A, d, h)$ be a curved dg algebra; then $\xi \in A^{1}$ is called MC if $h+d \xi+\frac{1}{2}[\xi, \xi]=0$.

Remark 4.4. The twisting of a curved dg algebra by an $\mathrm{MC}$ element is an uncurved algebra.

Example 4.5. (1) Let $A$ be a unital dg algebra, then $B A:=\hat{T} \Sigma\left(A^{*}\right)$ is an (uncurved) acyclic local pseudo-compact algebra. Tensoring it with $A$ we get another dg algebra (although not pseudo-compact unless $A$ is finite 
dimensional): $C=B A \otimes A$. The algebra $C$ has a canonical MC element; choosing a basis $\left\{e_{i}\right\}$ in $A$ and the dual basis $\left\{e^{i}\right\}$ in $\Sigma\left(A^{*}\right)$ it is:

$$
\xi:=\sum e^{i} \otimes e_{i} \in B A \otimes A .
$$

Then $(B A \otimes A)^{\xi}$ is isomorphic to $\operatorname{Hoch}(A, A)$, the (unreduced) Hochschild complex of $A$ with coefficients in itself. If $C$ is a (unital) $\operatorname{dg}$ algebra supplied with a dg algebra map $A \rightarrow C$ then $\operatorname{Hoch}(A, C)$ can similarly be constructed as an algebra twisting of $B A \otimes C$.

Furthermore, let $M$ be an $A$-bimodule. Then viewing $B A \otimes M$ as a bimodule over $B A \otimes A$ we can form the twisted module $(B A \otimes M)^{[\xi \otimes 1+1 \otimes \xi]}$. Here $\xi \otimes 1+1 \otimes \xi \in(B A \otimes A) \otimes(B A \otimes A)^{\mathrm{op}}$. This results in what normally is denoted by $\operatorname{Hoch}(A, M)$, the unreduced Hochschild complex of $A$ with coefficients in $M$; it is thus naturally a bimodule over $\operatorname{Hoch}(A, A)$.

(2) Let $A$ be as above and consider a fake augmentation $\xi: A \rightarrow \mathbf{k}$ as an element in $B A$. Twisting by $\xi$ gives a local curved pseudo-compact algebra $B A^{\xi}$ with curvature denoted by $w$.

The reduced bar-construction $\bar{B} A:=\hat{T} \Sigma\left((A / \mathbf{k})^{*}\right)$ is a (curved) subalgebra in $B A^{\xi}$; in fact there is an isomorphism $B A^{\xi} \cong \bar{B} A\langle\langle x\rangle\rangle$ mapping $\xi \mapsto x$, where $d x=x^{2}+w$.

In the case when $\xi$ is a genuine augmentation we have $w=0$ and the inclusion $\bar{B} A \hookrightarrow B A$ is a quasi-isomorphism as $B A$ is the coproduct (in the category of associative $\mathbf{k}$-algebras) of $\bar{B} A$ with an acyclic dg algebra.

(3) Let again $A$ be a unital dg algebra and consider the curved pseudo-compact algebra $\bar{B} A=\hat{T} \Sigma\left((A / \mathbf{k})^{*}\right)$. Let $A_{+}$be the kernel of the (fake) augmentation $\epsilon: A \rightarrow \mathbf{k}$. Then $(A / k)^{*}$ can be identified with $A_{+}^{*}$ and $\bar{B} A$ with $\hat{T} \Sigma\left(A_{+}^{*}\right)$. Thus, consider the curved dg algebra $\hat{T} \Sigma\left(A_{+}^{*}\right) \otimes A$. It has a canonical element $\xi=\sum e^{i} \otimes e_{i}$ where $e_{i}$ is a basis in $A_{+}$and $e^{i}$ is the dual basis in $\Sigma\left(A_{+}^{*}\right)$. It turns out to be an MC element so twisting by it results in an uncurved dg algebra $\left(\hat{T} \Sigma\left(A_{+}^{*}\right) \otimes A\right)^{\xi}$. The latter dg algebra is $\overline{\operatorname{Hoch}}(A, A)$, the reduced Hochschild complex of $A$ with coefficients in itself. If $C$ is a (unital) dg algebra supplied with a dg algebra map $A \rightarrow C$ then $\overline{\operatorname{Hoch}}(A, C)$ can similarly be constructed as an algebra twisting of $\bar{B} A \otimes C$.

If $M$ is an $A$-bimodule one can similarly form $\overline{\operatorname{Hoch}}(A, M)$ as a bimodule over $\overline{\operatorname{Hoch}}(A, A)$.

(4) Let $M$ be a left dg module over a dg algebra $A$ and consider $\operatorname{Hoch}(A, A)=$ $(B A \otimes A)^{\xi}$ as above and its left module $(B A \otimes M)^{[\xi]}$. This module looks similar to $\operatorname{Hoch}(A, M)$ but the differential is slightly different: one has to omit the last term in (3.1). The complex $(B A \otimes M)^{[\xi]}$ is acyclic and is the dual of the standard bar-resolution of $M^{*}$ as a right $A$-module.

Similarly we can form the dg algebra $\overline{\operatorname{Hoch}}(A, A)=(\bar{B} A \otimes A)^{\xi}$ and its left module $(\bar{B} A \otimes M)^{[\xi]}$, which can be identified with the dual reduced standard bar-resolution $M^{*}$ as a right $A$-module.

Proposition 4.6. Let $A$ be a curved pseudo-compact algebra and $\xi \in A^{1}$. Then the category of $A$-modules and the category of $A^{\xi}$-modules are Quillen equivalent. 
To an $A$-module $N$ we associate an $A^{\xi}$-module $M^{[\xi]}$ and to an $A^{\xi}$-module $N$ we associate the module $N^{[-\xi]}$.

Proof. Clearly the categories are isomorphic: the $A$-module $\left(M^{[\xi]}\right)^{[-\xi]}$ is equal to $M$ and similarly with an $A^{\xi}$-module $N$.

As both the categories are of pseudo-compact modules, cofibrations are characterized as the injective morphisms with projective cokernels (disregarding differentials). Clearly this property is preserved by both the functors described in the proposition.

\section{Koszul duality}

Let $A$ be a unital dg algebra and $B:=\bar{B} A$ be its reduced bar-construction, a curved local pseudo-compact dg algebra.

Definition 5.1. We define a pair of (contravariant) functors between the categories of $A$-modules and of $B$-modules as follows. The functor $F$ associates to an $A$-module $N$, the twisted module:

$$
F(N)=\left(B \otimes N^{*}\right)^{[\xi]} .
$$

Here we view $B \otimes N^{*}$ as a $B \otimes B^{\mathrm{op}} \otimes A^{\mathrm{op}}$-module and $\xi$ is the canonical element in $B^{\mathrm{op}} \otimes A^{\mathrm{op}}$. Thus, $\left(B \otimes N^{*}\right)^{[\xi]}$ becomes a $B \otimes\left(B^{\mathrm{op}} \otimes A^{\mathrm{op}}\right)^{\xi}=B \otimes \overline{\operatorname{Hoch}}(A, A)^{\mathrm{op}}$ module and forgetting the $\overline{\operatorname{Hoch}}(A, A)^{\text {op }}$-action we get a $B$-module.

The functor $G$ in the opposite direction associates to a $B$-module $L$ the twisted module:

$$
G(L)=\left(A \otimes L^{*}\right)^{[\xi]}
$$

Here we view $A \otimes L^{*}$ as a $B^{\mathrm{op}} \otimes A^{\mathrm{op}} \otimes A=\bar{B} A^{\mathrm{op}} \otimes A^{\mathrm{op}} \otimes A$-module; $\xi$ is the canonical element in $\bar{B} A^{\mathrm{op}} \otimes A^{\mathrm{op}}$. Thus, $\left(A \otimes L^{*}\right)^{[\xi]}$ is a $\left(\bar{B} A^{\mathrm{op}} \otimes A^{\mathrm{op}}\right)^{\xi} \otimes A=$ $\overline{\operatorname{Hoch}}(A, A)^{\mathrm{op}} \otimes A$-module and forgetting the $\overline{\operatorname{Hoch}}(A, A)^{\mathrm{op}}$-action we get an $A$ module.

Explicitly then, if $\alpha \in F(N)$ is a map $A^{\times k} \times N \rightarrow \mathbf{k}$, then $d \alpha$ maps:

$$
\begin{aligned}
\left(a_{1}, \cdots, a_{k+1}, n\right) \mapsto \quad & \epsilon\left(a_{1}\right) \alpha\left(a_{2}, \cdots, a_{k+1}, n\right) \\
- & \alpha\left(a_{1} a_{2}, \cdots, a_{k+1}, n\right) \\
+ & \cdots \\
+ & (-1)^{k+1} \alpha\left(a_{1}, \cdots, a_{k+1}, n\right),
\end{aligned}
$$

where as before $\epsilon$ denotes a fake augmentation and the elements $a_{i}, i=1, \ldots, k+1$ are understood to be even, as before. It is clear that $d^{2}$ induces application of the curvature element in $B$ rather than commutation with it, so $F(N)$ is indeed a $B$-module.

From [7, Theorem 6.3a] we know that $F, G$ form a mutually inverse pair of equivalences of categories. This equivalence is then a Quillen equivalence with respect to the closed model category structures on $A$-modules and $B$-modules. Thus we have:

Theorem 5.2. The functors $F, G$ form a (contravariant) Quillen equivalence between the categories of $A$-modules and $B$-modules. 
Remark 5.3. (1) The action of $\overline{\operatorname{Hoch}}(A, A)$ was not mentioned in [7] and the functors $F$ and $G$ were described in a different, although equivalent, language. The role of the additional right action of $\overline{\operatorname{Hoch}}(A, A)$ is not completely clear. One can speculate that $\operatorname{since} \overline{\operatorname{Hoch}}(A, A)$ is quasi-isomorphic to a strictly commutative dg algebra, its left and right actions coincide in some strong homotopy sense and moreover, are formal consequences (again in an appropriate strong homotopy sense) of the actions of $A$ or $B$.

(2) The action of the curved dg algebra $B$ on $F(N)$ is obtained as a restriction using the inclusion of curved dg algebras $B \hookrightarrow B \otimes \overline{\operatorname{Hoch}}(A, A): b \mapsto b \otimes 1$. Note that this required the twist: the corresponding map $B \rightarrow B \otimes B^{\mathrm{op}} \otimes$ $A^{\text {op }}$ is not a map of curved dg algebras and $B \otimes N^{*}$ is not a $B$-module, just as $B$ is not a $B$-module.

\section{Covariant Morita Equivalence for modules over A PSEUDO-COMPACT ALGEBRA}

Let $B$ be a pseudo-compact curved dg algebra and $M$ be a non-zero finitedimensional vector space over $\mathbf{k}$. Then $E^{\prime}:=B \otimes$ End $M$ is a curved dg pseudocompact algebra. We have:

Proposition 6.1. The categories of $B$-modules and of $E^{\prime}$-modules are Quillen equivalent. To a $B$-module $N$ we associate the $E^{\prime}$-module $F^{\prime}(N):=N \otimes M$. To an $E^{\prime}$-module $L$ we associate a $B$-module $G^{\prime}(L):=\operatorname{Hom}_{E}(B \otimes M, L) \cong$ $\operatorname{Hom}_{\text {End } M}(M, L)$.

Proof. We have natural isomorphisms:

$$
\begin{aligned}
G^{\prime} F^{\prime}(N) & =\operatorname{Hom}_{\operatorname{End} M}(M, N \otimes M) \\
& \cong \operatorname{Hom}_{\operatorname{End} M}(M, M) \otimes N \cong \mathbf{k} \otimes N=N, \\
F^{\prime} G^{\prime}(L) & =\operatorname{Hom}_{\operatorname{End} M}(M, L) \otimes M \\
& \cong \operatorname{Hom}\left(M^{*}, \operatorname{Hom}_{\operatorname{End} M}(M, L)\right) \\
& \cong \operatorname{Hom}_{\operatorname{End} M}\left(M^{*} \otimes M, L\right) \\
& \cong \operatorname{Hom}_{\operatorname{End} M}(\operatorname{End} M, L)=L .
\end{aligned}
$$

It remains to verify that $F^{\prime}$ preserves cofibrations and that $G^{\prime}$ preserves fibrations. As a module over itself End $M$ is a direct sum of copies of $M$. So $M$ is a projective End $M$ module.

Thus given a surjective map $f: L_{1} \rightarrow L_{2}$, the induced map $G(f)$ is surjective, as we may lift any element $g \in \operatorname{Hom}_{\operatorname{End} M}\left(M, L_{2}\right)$ :

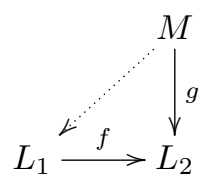

Thus $G^{\prime}$ preserves fibrations. 
A cofibration of $B$-modules $f: N_{1} \rightarrow N_{2}$ is characterized by being part of an exact sequence:

$$
0 \rightarrow N_{1} \stackrel{f}{\rightarrow} N_{2} \rightarrow P \rightarrow 0,
$$

for some $B$-module that is projective as a graded $B$-module, forgetting differentials. Applying $F^{\prime}$ we obtain the exact sequence:

$$
0 \rightarrow N_{1} \otimes M \stackrel{f \otimes 1_{M}}{\rightarrow} N_{2} \otimes M \rightarrow P \otimes M \rightarrow 0 .
$$

To deduce that $F^{\prime}$ preserves cofibrations we need only note that $P \otimes M$ is a summand of the projective module $E^{\prime}$-module $P \otimes$ End $M$.

\section{Composing the equivalences}

We now have all the ingredients to prove our main theorem. We have the following chain of Quillen equivalences:

$$
A \text {-mod } \leftrightarrow(\bar{B} A \text {-mod })^{\text {op }} \leftrightarrow(\bar{B} A \otimes \text { End } M \text {-mod })^{\text {op }} .
$$

Note that both $\bar{B} A$ and $\bar{B} A \otimes$ End $M$ are curved pseudo-compact algebras. Using Example 4.5 (3) we recognize $\overline{\operatorname{Hoch}}(A$, End $M)$ as an appropriate twisting $(\bar{B} A \otimes \text { End } M)^{\xi}$ and using Proposition 4.6 conclude that $E^{\prime}:=\bar{B} A \otimes$ End $M-$ modules are Quillen equivalent to $E:=\overline{\operatorname{Hoch}}(A$, End $M)$-modules.

Moreover, the composition of functors from left to right is clearly $F$ as defined in Theorem 2.1. This completes the proof of Theorem 2.1.

Tracing the functors going in the opposite direction we can give the following explicit description of the inverse equivalence $(E \text {-mod })^{\text {op }} \rightarrow A$-mod. Let $L$ be in $E$-mod. Considering the canonical element $\xi$ in $E$ we have $E^{-\xi}=\bar{B} A \otimes$ End $M$ and so $L^{[-\xi]}$ is a module over the curved dg algebra $\bar{B} A \otimes$ End $M$.

Applying $G^{\prime}$ returns the $\bar{B} A$ module $\operatorname{Hom}_{\operatorname{End} M}\left(M, L^{[-\xi]}\right)$. In order to dualize this we employ the following lemma.

Lemma 7.1. Given an End $M$-module $K$ we have: $\operatorname{Hom}_{\operatorname{End} M}(M, K)^{*} \cong \operatorname{Hom}_{\operatorname{End} M}(K, M)$.

Proof. Replacing $B$ in Proposition 6.1 with $\mathbf{k}$ gives us the Morita duality between $\mathbf{k}$ and End $M$ :

$$
\operatorname{Hom}_{\text {End } M}(K, M) \cong \operatorname{Hom}_{\mathbf{k}}\left(\operatorname{Hom}_{\operatorname{End} M}(M, K), \mathbf{k}\right) \cong \operatorname{Hom}_{\text {End } M}(M, K)^{*} .
$$

Thus applying $G$ to $\operatorname{Hom}_{\operatorname{End} M}\left(M, L^{[-\xi]}\right)$, we get a twist of the $A \otimes A^{\mathrm{op}} \otimes \bar{B} A^{\mathrm{op}}$ module:

$$
A \otimes \operatorname{Hom}_{\text {End } M}\left(L^{[-\xi]}, M\right) .
$$

Applying this (un)twist, we obtain that $\left(A \otimes \operatorname{Hom}_{\operatorname{End} M}\left(L^{[-\xi]}, M\right)\right)^{[\eta]}$ is a module over $A \otimes\left(A^{\mathrm{op}} \otimes \bar{B} A^{\mathrm{op}}\right)^{\eta}=A \otimes \overline{\operatorname{Hoch}}(A, A)^{\mathrm{op}}$ (where $\eta$ is the corresponding $\mathrm{MC}$ element). Finally, forgetting the action of $\overline{\operatorname{Hoch}}(A, A)$, we obtain an $A$ module $\left(A \otimes \operatorname{Hom}_{\operatorname{End} M}\left(L^{[-\xi]}, M\right)\right)^{[\eta]}$. This is the value of our composite functor $(E \text {-mod })^{\text {op }} \rightarrow A$-mod on $L$. 


\section{Equivalences Between CATEgories of Modules AND PSEUdo-Compact MODULES}

We saw that the category of modules over a dg algebra is always Quillen equivalent to some category of pseudo-compact modules (Corollary 2.3). It is natural to ask when, conversely, the category of pseudo-compact modules over a pseudocompact dg algebra is Quillen equivalent to the category of modules over some $\mathrm{dg}$ algebra. The following result gives an answer to that question; here and later on $D(A)$ and $D(B)$ stand for the derived categories of $A$-modules and of pseudocompact $B$-modules respectively, i.e. the homotopy categories of the corresponding closed model structures. The category $D(B)$ is the derived category of the second kind; even when $B$ is finite dimensional, it does not coincide, in general, with its usual derived category (more on that later).

Theorem 8.1. Let $B$ be a pseudo-compact $d g$ algebra. The following are equivalent:

(1) There exists a dg algebra $A$ and an equivalence $F: D(A) \cong D(B)^{\mathrm{op}}$,

(2) $D(B)^{\text {op }}$ admits a compact generator,

(3) $D(B)^{\text {op }}$ admits a finite-dimensional (necessarily compact) generator.

If any of these conditions holds then in fact there exists a $d g$ algebra $A$ and a Quillen equivalence between $A$-mod and $(B \text {-mod) })^{\mathrm{op}}$.

In case $B=B^{0}$, i.e. $B$ is an ordinary pseudo-compact algebra, any of the three conditions above is equivalent to the following statement:

(4) There are finitely many isomorphism classes of simple (non- $d g$ ) B-modules.

Lemma 8.2. Let $B$ be pseudo-compact $d g$ algebra. Then $D(B)^{\text {op }}$ is compactly generated, and an object of $D(B)^{\mathrm{op}}$ is compact if and only if it is in the thick subcategory of $D(B)^{\text {op }}$ generated by (totally) finite-dimensional modules.

Proof. By $[7, \S 5.5]$, the finite-dimensional $B$-modules form a set of compact objects that generate $D(B)^{\mathrm{op}}$. It follows that the compact objects in $D(B)^{\text {op }}$ are generated as a thick subcategory of $D(B)^{\text {op }}$ by the finite-dimensional modules $[3$, Theorem 2.1.3(c)].

Proof of Theorem 8.1. Given an equivalence $F$, we have that $F(A)$ is a compact generator of $D(B)^{\text {op }}$. Hence, by Lemma 8.2, $F(A)$ is obtained from a finite set of totally finite-dimensional $B$-modules by a sequence of shifts, extensions and retractions. Thus the direct sum of these finite-dimensional modules is a compact generator of $D(B)^{\mathrm{op}}$. This proves that (1) implies (3), and (3) obviously implies (2).

Now suppose (2) holds: let $N$ be a compact generator of $D(B)^{\mathrm{op}}$. Without loss of generality we may assume $N$ is cofibrant. Define a $\operatorname{dg}$ algebra $A:=\operatorname{End}_{B}(N)$. Then the functor

$$
F(-)=\operatorname{Hom}_{A}(-, N): A-\bmod \rightarrow(B-\bmod )^{\mathrm{op}}
$$

is left adjoint to the functor

$$
G(-)=\operatorname{Hom}_{B}(-, N):(B-\bmod )^{\mathrm{op}} \rightarrow A-\bmod .
$$


Recall [7] that cofibrations in $A$-mod are the injective maps with cofibrant (in particular projective) cokernel, whereas fibrations are the surjective maps. Similarly fibrations in $(B \text {-mod })^{o p}$ are the injective maps with projective cokernel, and fibrations are the surjective maps. It is then easy to see that $F$ preserves cofibrations and $G$ preserves fibrations. Moreover $F(A) \cong N$ and $G(N) \cong A$. Since $A$ and $N$ are compact generators in $D(A)$ and $D(B)$ respectively, it follows that $F$ and $G$ induce inverse equivalences of $D(A)$ and $D(B)^{\mathrm{op}}$.

Suppose now that $B$ is an ordinary pseudo-compact algebra. Simple (non-dg) $B$-modules may be regarded as simple dg $B$-modules concentrated in degree 0 , and any simple dg $B$-module arises in this way, up to grading shift. If $B$ has finitely many isomorphism classes of simple modules, their direct sum is then a compact generator, as any finite-dimensional dg $B$-module has a finite composition series.

Conversely suppose $B$ has a finite-dimensional (compact) generator $N$. It suffices to show that any simple $B$-module $S$ is a composition factor of the cohomology $H(N)$, regarded as a (finite-dimensional) ungraded $B$-module. To confirm the latter, note that $S$, as a compact object in $D(B)^{\mathrm{op}}$, is contained in the thick subcategory of $D(B)^{\mathrm{op}}$ generated by $N$, and observe that shifts, retracts and extensions of $\operatorname{dg} B$-modules cannot create new composition factors in cohomology.

Remark 8.3. The obvious generalisation of condition (4) in Proposition 8.1 to arbitrary pseudo-compact dg algebras $B$ would be the following:

(4') There are finitely many isomorphism classes of simple $\mathrm{dg} B$-modules, up to grading shift.

We do not know whether Proposition 8.1 holds in this generality. As a case in point, the pseudo-compact dg algebra $E=\overline{\operatorname{Hoch}}^{\bullet}(A$, End $M)$ of Theorem 2.1 has a unique simple ( $\mathrm{dg}$ ) module up to isomorphism and grading shift - the module $M$.

Remark 8.4. Let $G$ be an affine group over a field $\mathbf{k}$; denote its coordinate ring by $\mathbf{k}[G]$. The category of $G$-modules may be identified with the category of $\mathbf{k}[G]$ comodules, or equivalently, with the category of modules over the pseudo-compact algebra $\mathbf{k}[G]^{*}$. Thus it is natural to consider the derived category $D\left(\mathbf{k}[G]^{*}\right)$ and to ask for which $G$ it admits a compact generator. By Theorem 8.1 this holds if and only if there are finitely many isomorphism classes of simple $G$-modules.

In case $G$ is a smooth affine algebraic group over an algebraically closed field, we can give the following answer: $G$ has finitely many simple modules if and only if $G^{0}$, the connected component of the identity, is unipotent. Indeed, since $G^{0}$ is a normal subgroup of finite index in $G$, the standard arguments of Clifford theory imply that $G$ has finitely many simple modules precisely when $G^{0}$ does. If $G^{0}$ is unipotent it has a unique simple module. On the other hand if $G^{0}$ is not unipotent, it has a nontrivial reductive quotient and thus infinitely many isomorphism classes of simple modules.

\section{Comparison with Classical Morita duality}

Our main result, Theorem 2.1, is a Quillen anti-equivalence, or duality between two module categories; it is given as a kind of derived Hom functor. This suggests 
a close relationship with Morita duality [6,5] which also studies contravariant equivalences between various categories of modules. We will see that that our result can indeed be viewed as an extension of Morita duality in the case when the algebra in question is finite dimensional and of finite global dimension.

Let us first present a kind of derived Morita duality when $A$ is an ordinary (i.e. non-dg) finite-dimensional algebra; this will be our standing assumption in this section. If $M$ is a finite-dimensional injective cogenerator of the category of $A$-modules and $\Gamma:=\operatorname{End}_{A}(M)$ then the category of finite-dimensional $A$-modules is anti-equivalent to the category of finite-dimensional $\Gamma$-modules via the functors $F: N \mapsto \operatorname{Hom}_{A}(N, M)$ and $G: L \mapsto \operatorname{Hom}_{\Gamma}(L, M)$ (see e.g. [9, Theorem 7.11]). Note that since $\Gamma$ is a finite dimensional algebra, it makes sense to consider the category of its (left) pseudo-compact modules which we will denote by $\Gamma^{\mathrm{ps}}-$ mod; note that its opposite category is naturally identified with the category of $\Gamma^{*}$ comodules. The following result is an easy extension of this version of Morita duality.

Theorem 9.1. The functors $F$ and $G$ determine an anti-equivalence between the abelian categories $A-$ mod and $\Gamma^{\mathrm{ps}}-$ mod.

Proof. The functor $G: \Gamma^{\mathrm{ps}}-\bmod \rightarrow A-\bmod$ can be factored as a composition,

$$
\Gamma^{\mathrm{ps}} \text {-mod } \longrightarrow \Gamma \text {-mod } \longrightarrow A \text {-mod, }
$$

as follows:

$$
L \mapsto L^{*} \mapsto \operatorname{Hom}_{\Gamma}\left(M^{*}, L^{*}\right) \cong \operatorname{Hom}_{\Gamma}(L, M),
$$

where $L$ is a pseudo-compact $\Gamma$-module. The functor of linear duality $L \rightarrow L^{*}$ is clearly an anti-equivalence between $\Gamma^{\mathrm{ps}}-\bmod$ and $\Gamma-\bmod$ whereas the functor $\operatorname{Hom}_{\Gamma}\left(M^{*},-\right)$ is the usual covariant Morita equivalence between $\Gamma-\bmod$ and $A$ $\bmod \left(\right.$ note that $M^{*}$ is a projective generator of $A-\bmod$ since $M$ is an injective cogenerator).

Remark 9.2. It follows that $F$ and $G$ determine an anti-equivalence between the homotopy categories of complexes in $A$-mod and $\Gamma^{\mathrm{ps}}$-mod. Taking the Verdier quotient by the acyclic complexes, we conclude that $D(A)$, the derived category of $A$, is anti-equivalent to $D^{I}\left(\Gamma^{\mathrm{ps}}\right)$, the derived category of pseudo-compact $\Gamma$ modules of the first kind (cf. [7] concerning this terminology). Note that our previous results were concerned with the derived categories of pseudo-compact modules of the second kind.

Recall from [7] that a complex of $A$-modules is absolutely acyclic if it belongs to the minimal thick subcategory of the category $\operatorname{Hot}(A$-mod) of $A$-modules up to homotopy, containing acyclic bounded complexes. The Verdier quotient of Hot $(A$-mod $)$ by the subcategory of absolutely acyclic complexes is called the $a b$ solute derived category of $A$ and denoted by $D^{\text {abs }}(A)$. In the same way we can define the absolute derived category $D^{\text {abs }}\left(\Gamma^{\mathrm{ps}}\right)$ of pseudo-compact $\Gamma$-modules. It follows similarly that the functors $F$ and $G$ determine an anti-equivalence between $D^{\mathrm{abs}}(A)$ and $D^{\mathrm{abs}}\left(\Gamma^{\mathrm{ps}}\right)$.

We observe that there is a simple criterion for the two types of derived categories to coincide. 
Lemma 9.3. If a finite-dimensional algebra $\Gamma$ has finite global dimension then the following categories coincide:

(1) $D^{\mathrm{abs}}(\Gamma)$ and $D(\Gamma)$;

(2) $D^{\mathrm{abs}}\left(\Gamma^{\mathrm{ps}}\right), D\left(\Gamma^{\mathrm{ps}}\right)$ and $D^{I}\left(\Gamma^{\mathrm{ps}}\right)$.

Proof. Note first of all that $(1) \Longrightarrow(2)$ by Remark 9.2. Thus, it suffices to prove (1). To this end let $M$ be an acyclic complex of $\Gamma$-modules; we have to show that $\Gamma$ is absolutely acyclic. Let $M\langle n, m\rangle$ be the complex of $\Gamma$-modules such that:

$$
M\langle n, m\rangle^{i}=\left\{\begin{array}{l}
M^{i} \text { if } n<i<m \\
\operatorname{ker} d: M^{n} \rightarrow M^{n+1} \text { if } i=n \\
\text { Coker } d: M^{m-1} \rightarrow M^{m} \text { if } i=m \\
0 \text { if } i<n \text { or } i>m
\end{array}\right.
$$

Then clearly $M\langle n, m\rangle$ is absolutely acyclic and

$$
M \simeq \operatorname{holim}_{n} \operatorname{hocolim}_{m} M\langle n, m\rangle .
$$

It follows by [7, Theorem 3.6] that the category of absolutely acyclic complexes is closed with respect to arbitrary direct sums and direct products (this is where the finite global dimension assumption is used), and therefore also with respect to homotopy limits and colimits along directed systems . Therefore $M$ is absolutely acyclic.

Remark 9.4. If $\Gamma$ is a pseudo-compact dg algebra of finite global dimension then Positselski [7, Theorem 3.6 and 4.5] showed that various derived categories of second kind of $\Gamma$ coincide. The result above shows that under the additional assumption that $\Gamma$ is concentrated in degree zero, these derived categories of second kind also coincide with the ordinary derived categories.

We can now formulate the main result of this section.

\section{Theorem 9.5.}

(1) Let $A$ be a finite-dimensional algebra of finite global dimension. Then the categories of $d g A$-modules and of pseudo-compact $d g A$-modules are Quillen anti-equivalent.

(2) Conversely, suppose that there exists a finite-dimensional algebra $\Gamma$ such that the categories $D(A)$ and $D\left(\Gamma^{\mathrm{ps}}\right)^{\mathrm{op}}$ are equivalent. Then $A$ and $\Gamma$ both have finite global dimension, and $D(A)$ and $D(\Gamma)$ are equivalent.

Proof. Note that the k-linear duality functor $L \mapsto L^{*}$ determines an anti-equivalence between the abelian categories $A$-mod and $A^{\mathrm{ps}}$-mod; it is also a Quillen functor between the corresponding closed model categories. Now the homotopy category of $A$-mod (in the sense of closed model categories) is the derived category $D(A)$. By Lemma 9.3 the homotopy category of the closed model category $A^{\mathrm{ps}}-$ mod is the same as $D^{I}\left(A^{\mathrm{ps}}\right)$ which is then equivalent to $D(A)$ under the functor of linear duality. This proves (1).

Now suppose that $D(A)$ and $D\left(\Gamma^{\mathrm{ps}}\right)$ are equivalent. Then arbitrary products and coproducts exist in $D\left(\Gamma^{\mathrm{ps}}\right)$. Since absolutely acyclic pseudo-compact $\Gamma$-modules vanish in $D\left(\Gamma^{\mathrm{ps}}\right)$, arguing as in the proof of Lemma 9.3 we see, that 
the same is true of all acyclic pseudo-compact $\Gamma$-modules. Thus $D\left(\Gamma^{\mathrm{ps}}\right)$ coincides with $D^{I}\left(\Gamma^{\mathrm{ps}}\right)$, and is thus anti-equivalent to $D(\Gamma)$ via linear duality. Now recall from Lemma 8.2 that finite-dimensional $\Gamma$-modules are compact objects in $D\left(\Gamma^{\mathrm{ps}}\right)^{\mathrm{op}}$. We deduce from the equivalence $D(\Gamma) \cong D\left(\Gamma^{\mathrm{ps}}\right)^{\mathrm{op}}: M \mapsto M^{*}$ that any finite-dimensional dg $A$-module is compact in $D(A)$, which implies that $A$ has finite global dimension, and therefore so does $\Gamma$.

Remark 9.6. For a finite-dimensional algebra $A$ there are two natural choices for a finite-dimensional module $M$; namely one can take $M=A$ or $M=A^{*}$; the latter choice having the advantage of being an injective cogenerator. Then Theorem 2.1 states that the category $A-\bmod$ and $E-\bmod$ where $E:=\overline{\operatorname{Hoch}}(A, \operatorname{End}(M))$ are Quillen equivalent. Since $E$ is quasi-isomorphic to $\operatorname{RHom}_{A}(M, M)$, for $M=A$ or $M=A^{*}$ it is further quasi-isomorphic to $A$. One can ask whether the above equivalence simplifies to a Quillen equivalence between $A$-modules and pseudocompact $A$-modules. Theorem 9.5 says, in particular, that this is the case only when $A$ has finite global dimension.

\section{REFERENCES}

[1] A. Beilinson, V. Ginzburg, W. Soergel, Koszul duality patterns in representation theory. J. Amer. Math. Soc. (2) 9 (1996) 473-527.

[2] J. Chuang \& A. Lazarev, $L_{\infty}$ maps and twistings. Homology Homotopy Appl. (2) 13 (2011) $175-195$.

[3] M. Hovey, J.H. Palmieri \& N.P. Strickland, Axiomatic stable homotopy theory. Mem. Amer. Math. Soc. 128 (1997), no. 610.

[4] B. Keller, Koszul duality and coderived categories (after K. Lefèvre). October 2003. Available from http://www.math.jussieu.fr/ keller/publ/index.html.

[5] T.Y. Lam, Lectures on modules and rings. Graduate Texts in Mathematics, 189. SpringerVerlag, New York 1999.

[6] K. Morita, Duality for modules and its applications to the theory of rings with minimum condition. Sci. Rep. Tokyo Kyoiku Daigaku 6 (1958) 83-172.

[7] L. Positselski, Two kinds of derived categories, Koszul duality, and comodule-contramodule correspondence. Mem. Amer. Math. Soc. 212 (2011), no. 996.

[8] J.P. Pridham, Tannaka duality for enhanced triangulated categories. arXiv:1309.0637, http://arxiv.org/abs/1309.0637

[9] A. Skowroński, K. Yamagata, Frobenius algebras. I. Basic representation theory. EMS Textbooks in Mathematics. European Mathematical Society, Zürich 2011.

[10] M. Takeuchi, Morita theorems for categories of comodules. J. Fac. Sci. Univ. Tokyo. 24 (1977), 1483-1528.

[11] M. Van den Bergh, Calabi-Yau algebras and superpotentials. Selecta Math. 21 (2015) 555603. 